\title{
TRIAGEM AUDITIVA: CONCORDÂNCIA ENTRE OS MÉTODOS COMPORTAMENTAL E OBJETIVO
}

\section{Hearing screening: agreement among behavioral and objective methods}

\author{
Bruna Machado Correa $^{(1)}$, Larissa Lautenschlager ${ }^{(2)}$, Tania Tochetto(3), \\ Luciane da Costa Pacheco(4), Celina Rech Maggi(5), Maiara Santos Gonçalves ${ }^{(6)}$
}

\section{RESUMO}

Objetivo: verificar a concordância entre os resultados da triagem auditiva por meio de observação de respostas comportamentais e de emissões otoacústicas evocadas transientes. Método: foi realizado estudo clínico duplo-cego com 139 crianças de um a 180 dias de vida, atendidas no serviço de Triagem Auditiva Neonatal do Hospital Universitário de Santa Maria. Diferentes examinadores verificaram a presença do reflexo cócleo-palpebral e de emissões otoacústicas evocadas transientes. Resultados: das 139 crianças avaliadas, 123 apresentaram reflexo cócleo-palpebral e emissões otoacústicas; 10 apresentaram somente reflexo cócleo-palpebral; três apresentaram somente emissões otoacústicas; três não apresentaram respostas em ambos os testes. Dezesseis crianças deveriam ser retestadas. Nove não compareceram. Das sete crianças retestadas, duas passaram em ambos os testes e cinco mantiveram falha e foram encaminhadas para avaliação de Potencial Evocado Auditivo de Tronco Encefálico. Uma delas não compareceu. Duas crianças tiveram emissões otoacústicas presentes e reflexo cócleo-palpebral ausente. Na avaliação de Potencial Evocado Auditivo de Tronco Encefálico uma delas manifestou audição normal e outra perda auditiva profunda. Uma criança que não evidenciou emissões otoacústicas, mas manifestou reflexo cócleo-palpebral, teve Potencial Evocado Auditivo de Tronco Encefálico compatível com perda auditiva moderada bilateral. A criança que falhou em ambos os testes apresentou perda auditiva severa bilateral na avaliação de Potencial Evocado Auditivo de Tronco Encefálico. Conclusão: a presença de emissões otoacústicas concomitante com ausência de reflexo cócleo-palpebral pode ser sinal de neuropatia auditiva. A análise de emissões otoacústicas e a avaliação do reflexo cócleo-palpebral são procedimentos complementares. A aparente discordância entre alguns resultados pode apontar diferentes tipos de comprometimento auditivo.

DESCRITORES: Audição; Triagem Neonatal; Diagnóstico Precoce; Criança

(1) Fonoaudióloga; formada pela Universidade Federal de Santa Maria, UFSM, Santa Maria, RS, Brasil; Mestre em Distúrbios da Comunicação Humana pela Universidade Federal de Santa Maria.

(2) Fonoaudióloga, formada pela Universidade Federal de Santa Maria, UFSM, Santa Maria, RS, Brasil; Mestre em Distúrbios da Comunicação Humana pela Universidade Federal de Santa Maria.

(3) Fonoaudióloga; Professora Doutora Associada do Departamento de Fonoaudiologia da Universidade Federal de Santa Maria, Santa Maria, RS, Brasil.

(4) Fonoaudióloga, formada pela Universidade Federal de Santa Maria, UFSM, Santa Maria, RS, Brasil; Mestre em Distúrbios da Comunicação Humana pela Universidade Federal de Santa Maria.

(5) Fonoaudióloga, formada pela Universidade Federal de Santa Maria, UFSM, Santa Maria, RS, Brasil; Mestre em Distúrbios da Comunicação Humana pela Universidade Federal de Santa Maria.

\section{INTRODUÇÃO}

Sabe-se que a saúde auditiva é fator determinante para a aquisição e o desenvolvimento da linguagem oral. As habilidades de organização perceptual, recepção e estruturação das informações, aprendizagem e interações sociais dependem de um bom desenvolvimento de linguagem. Dessa forma, audição e linguagem são funções correlacionadas e interdependentes ${ }^{1}$, sendo inquestionável

(6) Fonoaudióloga, formada pela Universidade Federal de Santa Maria, UFSM, Santa Maria, RS, Brasil; Mestre em Distúrbios da Comunicação Humana pela Universidade Federal de Santa Maria.

Conflito de interesses: inexistente 
que a perda auditiva infantil não detectada precocemente acarreta uma série de déficits à criança, à família e à sociedade.

Considerando os benefícios do diagnóstico precoce da deficiência auditiva infantil, as Academias Americanas de Audiologia, Otorrinolaringologia e Pediatria reunidas no Joint Committee on Infant Hearing ${ }^{2}$ recomendam a Triagem Auditiva Neonatal Universal (TANU) por meio de Emissões Otoacústicas desde 1994. O Comitê Brasileiro Sobre Perdas Auditivas na Infância ${ }^{3}$ sugere adicionar a observação do reflexo cócleo-palpebral (RCP) frente a estímulo sonoro.

Devido à inexistência de equipamento para registro de Emissões Otoacústicas, a Triagem Auditiva Neonatal (TAN) vinha sendo feita no Hospital Universitário de Santa Maria (HUSM) somente com observação de respostas comportamentais. Com a aquisição do analisador coclear surgiu a possibilidade de aplicar a TAN tal como recomendada pelo Joint Committee on Infant Hearing ${ }^{2,4}$ e pelo Comitê Brasileiro Sobre Perdas Auditivas na Infância ${ }^{3}$.

A observação de respostas comportamentais permite determinar alterações centrais, porém tem limites, tais como não identificar perdas auditivas leves, moderadas e unilaterais ${ }^{5}$. Por outro lado, a análise de emissões otoacústicas não possibilita avaliar o funcionamento auditivo central, mas é um procedimento objetivo, mais preciso e reduz a influência do examinador na interpretação dos resultados, além de revelar a presença de alterações periféricas ${ }^{6}$.

O objetivo deste trabalho foi verificar a concordância entre os resultados obtidos na triagem auditiva por meio de observação de respostas comportamentais e de emissões otoacústicas evocadas transientes (EOE-t).

\section{MÉTODO}

As crianças participantes deste estudo foram atendidas no serviço de Triagem Auditiva Neonatal (TAN) do Hospital Universitário de Santa Maria (UFSM). Todos os pais/responsáveis pelas crianças concordaram que as mesmas participassem da pesquisa assinando o Termo de Consentimento Livre e Esclarecido.

Foram estudadas 139 crianças com idades entre um e 180 dias, nascidas no HUSM. Foi realizado um estudo clínico duplo cego em que foi verificada a presença de EOE-t e de RCP por diferentes examinadores. Na ocasião da aplicação dos procedimentos propostos a criança estava em sono leve.

A verificação da presença de RCP foi realizada com som não calibrado de intensidade aproximada a $90 \mathrm{~dB}$ produzido por agogô. Os estímulos sonoros foram apresentados a $15 \mathrm{~cm}$ da fontanela da criança, a qual estava posicionada em decúbito dorsal.

A captação das EOE-t foi realizada em local silencioso, com o aparelho Otoread Clínico da marca Interacoustics/Audiotest. A faixa de frequências foi de 500 a $4000 \mathrm{~Hz}$, a duração do estímulo sonoro foi de aproximadamente 64 segundos $e$ o nível de intensidade sonora do "click" foi de 83 dBNPS. As EOE-t foram consideradas presentes quando houve resposta para no mínimo três frequências, com relação sinal-ruído igual ou superior a $4 \mathrm{~dB}$.

As crianças que não apresentaram $\mathrm{RCP}$ e/ ou EOE-t foram retestadas. As que falharam pela segunda vez em um ou outro teste foram encaminhadas para avaliação do Potencial Evocado Auditivo de Tronco Encefálico (PEATE).

A avaliação do PEATE foi realizada com o aparelho Racia. Utilizou-se "click" alternado em 100 microsegundos, apresentado a 30 pps, iniciando na intensidade de $80 \mathrm{~dB}$ até alcançar o limiar auditivo. A extensão dos filtros foi de $100 \mathrm{a} 3000 \mathrm{~Hz}$, a janela de 20 milisegundos e o transdutor utilizado foi o fone TDH 49. Todas as respostas foram obtidas com o mínimo de 2000 repetições.

A classificação do grau de perda auditiva a partir do resultado da avaliação do PEATE baseou-se na recomendação do Bureau International d'Audio Phonologie - BIAP? .

Este estudo foi aprovado pelo Comitê de Ética em Pesquisa em Seres Humanos da Universidade Federal de Santa Maria sob processo no 0131.0.243.000-08.

Os resultados obtidos foram armazenados no Programa SPSS v11.5. Utilizou-se o Teste de Fischer com $p=0,011$ na primeira testagem, e $p=$ 1,00 no reteste. $O$ nível de significância utilizado foi de $5 \%$.

\section{RESULTADOS}

Das 139 crianças avaliadas, 123 apresentaram RCP e EOE-t; 10 apresentaram somente RCP; três apresentaram somente EOE-t e três falharam em ambos os testes (Tabela 1).

Constatou-se que o uso de EOE-t associada à pesquisa do RCP revelou maior índice de resultados "passa" do que qualquer um dos métodos utilizados de forma isolada $(p=0,011)$ (Tabela 1$)$.

Deveriam ser retestadas 16 crianças que não apresentaram RCP e/ou EOE-t, no entanto somente sete compareceram (Tabela 2), com um percentual de $56,25 \%(n=9)$ de evasão.

No reteste, o tratamento estatístico não revelou diferença estatisticamente significante entre os resultados obtidos com a aplicação dos dois 
procedimentos de forma associada ou isoladamente $(p=1,00)$. Isso se deve, provavelmente, ao pequeno tamanho da amostra no reteste $(n=7)$.

Das cinco crianças que mantiveram a falha no reteste e foram encaminhadas para avaliação de PEATE, quatro compareceram. Os resultados obtidos na avaliação de PEATE foram: audição normal, perda auditiva moderada bilateral, perda auditiva profunda bilateral e perda auditiva severa bilateral (Figura 1).

\begin{tabular}{|l|l|}
\hline Ocorrência de RCP e EOE-t & Resultados da avaliação de PEATE \\
\hline Somente RCP presente & Perda Auditiva Neurossensorial Bilateral de Grau Moderado \\
\hline Somente EOE-t presentes & Audição Normal em ambas as orelhas \\
\hline Somente EOE-t presentes & Perda Auditiva Neurossensorial Bilateral de Grau Profundo \\
\hline RCP e EOE-t ausentes & Perda Auditiva Neurossensorial Bilateral de Grau Severo \\
\hline
\end{tabular}

RCP: reflexo cócleo-palpebral

EOE-t: Emissões Otoacústicas Evocadas Transientes

PEATE: Potencial Evocado Auditivo de Tronco Encefálico

\section{Figura 1 - Resultado da análise de EOE-T, RCP e PEATE em quatro crianças}

\section{DISCUSSÃO}

A associação entre testes subjetivos e objetivos trouxe aos profissionais de audiologia benefícios na realização do diagnóstico precoce de alterações auditivas, considerando que a discordância entre os resultados destes procedimentos não caracteriza erro nos resultados, mas sim diferentes tipos de comprometimento auditivo, como neuropatia auditiva $^{8}$ ou perda auditiva neurossensorial de grau moderado ${ }^{9}$.

As EOE-t avaliam o sistema auditivo periférico, especificamente as células ciliadas externas. As EOA não quantificam a deficiência auditiva, porém detectam a sua ocorrência, uma vez que estão presentes quando há integridade coclear ${ }^{6}$. Já a observação de respostas comportamentais informa tanto em relação à acuidade auditiva, quanto ao processamento auditivo central, uma vez que está baseada na mudança de comportamento da criança após a estimulação auditiva ${ }^{5}$.

Na primeira testagem verificou-se que $16(11 \%)$ crianças não apresentaram resposta em um ou outro teste (Tabela 1). A ausência de EOE-t ocorreu em quatro $(2,9 \%)$ crianças que tinham cerca de 24 horas de vida. É possível atribuir a ausência de EOE-t à presença de vérnix caseoso no meato acústico externo ${ }^{10}$, resíduos, descamação epitelial, alterações na membrana timpânica, alterações de orelha média, não absorção do líquido amniótico, oclusão parcial ou total do meato acústico externo por resíduos até aproximadamente o sétimo dia de vida $^{11}$. Estes fatores podem ocasionar redução ou abolição do sinal sonoro no meato acústico externo.
O segundo dia de vida é tido como o momento mais apropriado para a triagem auditiva neonatal com EOE-t. Estudo comparativo entre as EOE-t obtidas de recém-nascidos durante o primeiro e o segundo dia de vida demonstrou melhora significantes das respostas após a 24 $4^{\mathrm{a}}$ hora de vida ${ }^{12}$. Outro fator que impede a captação e registro das EOE-t é a condição patológica da orelha média, que geralmente diminui a amplitude das emissões, como nos casos de otites, em que há uma atenuação resultante dos efeitos de massa ou rigidez no sistema ossicular $^{13}$.

Tabela 1 - Ocorrência de reflexo cócleopalpebral e emissões otoacúscticas evocadas transientes na primeira testagem

\begin{tabular}{lcc}
\hline & $\mathbf{N}$ & $\%$ \\
\hline RCP e EOE-t presentes & 123 & $89^{*}$ \\
Somente RCP & 10 & 7 \\
Somente EOE-t & 3 & 2 \\
RCP e EOE-t ausentes & 3 & 2 \\
\hline Total & 139 & 100 \\
\hline
\end{tabular}

$p=0,011^{*}$

RCP: reflexo cócleo-palpebral;

EOE-t: Emissões Otoacústicas Evocadas Transientes.

Das dezesseis crianças que deveriam ser retestadas (Tabela 2 ), nove não compareceram $(56,25 \%)$. Acredita-se em uma possível associação entre a evasão e condições sócio-econômico-culturais destas parturientes provenientes de maternidades públicas ${ }^{5}$. 
Tabela 2 - Ocorrência de reflexo cócleopalpebral e emissões otoacúscticas evocadas transientes no reteste

\begin{tabular}{llc}
\hline & $\mathbf{N}$ & $\%$ \\
\hline RCP e EOE-t presentes & 2 & 28,57 \\
Somente RCP & 1 & 14,29 \\
Somente EOE-t & 2 & 28,57 \\
RCP e EOE-t ausentes & 2 & 28,57 \\
\hline Total & 7 & 100 \\
\hline p $=1,00$ & \multicolumn{3}{l}{} \\
RCP: reflexo cócleo-palpebral; & \\
EOE-t: Emissões Otoacústicas Evocadas Transientes.
\end{tabular}

Uma criança que não evidenciou EOE-t, mas manifestou RCP, teve PEATE compatível com perda auditiva moderada ${ }^{7}$ em ambas as orelhas (Figura 1). Tais respostas na avaliação de EOE-t são observadas quando os limiares auditivos são inferiores ou iguais a $30 \mathrm{dBNA}{ }^{14}$. Já a presença de RCP descarta a possibilidade de perda auditiva neurossensorial severa e profunda bilateral. Além disso, sabe-se que a eliciação do RCP permite identificar alterações centrais, porém não revela perdas auditivas leves, moderadas e unilaterais ${ }^{5}$. Os achados das EOE-t e RCP, neste caso, são compatíveis com o diagnóstico de perda auditiva moderada, segundo a avaliação de PEATE (Figura 1).

A criança que falhou em ambos os testes (EOE-t e RCP) apresentou perda auditiva severa bilateral na avaliação de PEATE (Figura 1). De fato, é esperada a ausência de RCP e EOE-t em perdas auditivas severas e profundas bilaterais ${ }^{15}$.

O grau das perdas auditivas encontradas neste estudo variou de moderado a profundo. Na literatura consultada, as perdas auditivas neurossensoriais são predominantemente moderadas (52,7\% dos casos) e severas/profundas $(47,3 \%$ dos recém-nascidos) ${ }^{16}$.

No reteste, duas crianças (12,5\%) não apresentaram RCP, entretanto as EOE-t foram eliciadas (Tabela 2). Conforme o resultado da avaliação de PEATE, a primeira criança apresentou audição normal e a segunda perda auditiva profunda
(Figura 1). Considera-se que 0 resultado da avaliação comportamental da primeira criança foi falso positivo. A observação de respostas comportamentais pode gerar um elevado índice de resultados falso-negativos e falso-positivos devido à subjetividade do exame. Sendo assim, faz-se necessário associar esta a uma avaliação objetiva $^{14}$. A perda auditiva profunda, evidenciada no PEATE da segunda criança, é sugestiva de neuropatia auditiva, atualmente chamada de Espectro da Neuropatia Auditiva ${ }^{17}$. O Espectro da Neuropatia Auditiva é determinado pela perda da sincronia na condução nervosa. O local específico da lesão não é definido, mas pode acometer as células ciliadas internas, as sinapses entre as células ciliadas internas e o nervo auditivo (VIII par), o próprio nervo auditivo ou várias dessas estruturas ao mesmo tempo $^{18}$. Os achados na avaliação audiológica nestes casos são EOE-t presentes (função coclear normal) e PEATE ausente ou severamente comprometido ${ }^{19}$. Em estudo realizado com uma população de crianças com perda auditiva profunda, a prevalência de neuropatia auditiva foi de $0,94 \%{ }^{20}$.

Com este estudo confirmou-se perda auditiva em três crianças. Na ocasião do diagnóstico audiológico todas tinham menos de seis meses de idade e foram encaminhados para intervenção fonoaudiológica, atendendo, portanto às recomendações do Joint Committee on Infant Hearing ${ }^{4}$ e GATANU ${ }^{21}$.

\section{CONCLUSÃO}

Os resultados obtidos sugerem que: mesmo quando as EOE-t estão presentes, a ausência de RCP deve ser considerada motivo para avaliar PEATE, podendo ser sinal de neuropatia auditiva; crianças com EOE-t ausentes podem ter RCP presente, dependendo do grau da perda auditiva; a análise de EOE-t e a avaliação do RCP são procedimentos complementares; a aparente discordância entre alguns resultados pode apontar diferentes tipos de comprometimento auditivo. 


\begin{abstract}
Purpose: to check the agreement among the obtained results in hearing screening through the observation of behavioral responses and the transient evoked otoacoustic emissions. Method: a double-blind study was carried out with 139 infants attended in Neonatal Hearing Screening service of Santa Maria University Hospital. Different examiners evaluated the presence of eye blink reflex and transient evoked otoacoustic emissions. Results: among the 139 evaluated infants, 123 showed eye blink reflex and otoacoustic emissions; 10 showed only otoacoustic emissions; and 3 failed in both tests. Sixteen infants should be re-evaluated. Nine did not come back to repeat the tests. Seven children were re-evaluated, two passed in both tests; five maintained the failed status and they were referred to evaluation on Auditory Response Audiometry. Two children showed otoacoustic emissions, but did not have eye blink reflex. In the evaluation of Auditory Response Audiometry, one manifested normal hearing and the other showed acute hearing loss. One child who did not evidence otoacoustic emissions, but manifested eye blink reflex, had compatible Auditory Response Audiometry with moderate hearing loss in both ears. The infant who failed in both test showed severe bilateral hearing loss in the evaluation of Auditory Response Audiometry. Conclusion: even though the otoacoustic emissions are present, the absence of eye blink reflex should be considered a reason to evaluate the Auditory Response Audiometry, and this can be sign of auditory neuropathy. Considering that the analysis of otoacoustic emissions and the evaluation of eye blink reflex are complement procedures. The apparent disagreement among some results can show different types of hearing disorder.
\end{abstract}

KEYWORDS: Hearing; Neonatal Screening; Early Diagnosis; Child

\section{REFERÊNCIAS}

1. Gatto $\mathrm{Cl}$, Tochetto TM. Deficiência auditiva infantil: implicações e soluções. Rev CEFAC. 2007;9(1):110-5.

2. Joint Committee on Infant Hearing - Position Statement. ASHA. 1994;36:38-41.

3. Comitê Brasileiro sobre Perdas Auditivas na Infância - Recomendação 01/99. Jornal do Conselho Federal de Fonoaudiologia. Brasília, maio/ junho, 2000.

4. Joint Committee on Infant Hearing - Position Statement. ASHA. Pediatrics 2007; 120: 898-921.

5. Machado MS. Triagem auditiva neonatal universal: projeto piloto no Hospital Universitário de Santa Maria [monografia]. Santa Maria (RS): Universidade Federal de Santa Maria; 2001.

6. Jardim IS, Mata CG, Carvalho RMM. Emissões otoacústicas evocadas por estímulos transientes e potencial evocado auditivo de tronco encefálico automático na triagem auditiva neonatal. Einstein. 2008;6(3):253-61.

7. Bureau Internacional d'Audio Phonologie. Audiometric classification of hearing impairment: recommendation 02/1. [citado 2008 jun 10]. Disponível em: <http://www. biap.biapanglais/ rec021.eng.htm
8. Rondina C, Matas CG. Neuropatia auditiva: estudo de caso. Acta ORL. 2006;24(1):8-15.

9. Franceschi CM, Tochetto $T$, Lautenschlager $L$. Emissões otoacústicas transientes como método complementar no topodiagnóstico de perdas auditivas neurossensoriais. Arq. Int. Otorrinolaringol. 2010;14(1):50-3.

10. Dhawan R, Mathur N. An alternative strategy for UHS in tertiary hospitals, within a developing country, using transient evoked oto-acoustic emissions and brainstem evoked response audiometry. The J Laryngol \& Otology. 2007;121(7): 639-43.

11. Hall JW. Handbook of otoacoustic emissions. Singular Publishing Group. San Diego; 2000.

12. Del Buono ZG, Mininni $F$, Delvecchio $M$, Pannacciulli C, Mininni S. Neonatal hearing screening during the first and second day of life. Minerva Pediatr. 2005; 57: 167-72.

13. Couto CM, Carvalho RMM. O efeito das orelhas externa e média nas emissões otoacústicas. Rev Bras Otorrinolaringol. 2009;75(1):15-23.

14. Weber BA, Diefendorf A. Triagem auditiva neonatal. In: Musiek FE, Rintelmann WF. Perspectivas atuais em avaliação auditiva. São Paulo: Manole, 2001. p. 323-41.

15. Botelho FA, Bouzada MCF, Resende LM, Silva CFXCA, Oliveira EA. Triagem Auditiva em neonatos. Rev Med Minas Gerais. 2008;18(Supl 1):139-45. 
16. Garcia CFD, Isaac ML, Oliveira JAA. Emissão otoacústica evocada transitória: instrumento para detecção precoce de alterações auditivas em recém-nascidos a termo e pré-termo. Rev Bras Otorrinolaringol. 2002; 68:344-52.

17. Pearce, W; Martin, RL. On auditory neuropathy, aka auditory neuropathy spectrum. Nuts \& Bolts The Hearing Journal. 2009. 62(2): 38-9.

18. Pinotti KSA, Corazza MCA, Alcarás PAS. Avaliação eletrofisiológica do nervo auditivo em pacientes normo-ouvintes com ausência do reflexo estapediano. Arq. Int. Otorrinolaringol. 2009;13(4):386-93.
19. Anastasio ART, Alvarenga KF, Costa Filho OA. Eletrococleografia extratimpânica na neuropatia/ dessincronia auditiva. Rev Bras Otorrinolaringol. 2008;74(1):132-6.

20. Foerst A, Beutner D, Lang-Roth R, Huttenbrink $\mathrm{K}$, Wendel $\mathrm{H}$, Walger M. Prevalence of auditory neuropathy/synaptopathy in a population of children with profound hearing loss. Int $\mathrm{J}$ Otorhinolaryngol. 2006;70(8):1415-22.

21. Grupo de Apoio à Triagem Auditiva Neonatal Universal-Brasil (GATANU). [acesso em 2009 nov 12]. Disponível em: http://www.gatanu.org
http://dx.doi.org/10.1590/S1516-18462011005000051

RECEBIDO EM: 17/08/2010

ACEITO EM: 13/01/2011

Endereço para correspondência:

Maiara Santos Gonçalves

Av Evaldo Behr no 02, Parque Residencial Novo

Horizonte, Bairro Camobi

Santa Maria - RS

CEP: 97110-801

E-mail: maiarasg@yahoo.com.br 